\title{
Application of Dual DSP Target Tracking System Based on SVM
}

\author{
Xinghong $\mathrm{Li}^{1, \mathbf{a}}$, Qian Xiang ${ }^{2, \mathbf{b}}$, Jin Fa Cheng ${ }^{3, \mathbf{c}}$ \\ 1,2,3 The Engineering \& Technical college of Chengdu University of \\ Technology,Leshan,Sichuan,China \\ a545178279@qq.com, ${ }^{\mathrm{b}} 1139916444 @ q q . c o m,{ }^{\mathrm{c}} 798598367 @ q q . c o m$
}

Key words: Dual DSP, target tracking, the SVM, automatic detection

Abstract: With the real-time imaging tracking technology is widely used in various fields, military response to real-time tracking system,the precision and rapidity requirements are also increasing.This system for moving object in video monitoring field,designed target tracking system based on TMS320C6455 of dual DSP.At the same time, because the target rotating shade, dimension change,deformation and the change of light caused by low tracking precision of faults for the traditional target tracking system, the system applied the statistical learning theory of support vector machine (SVM) to the target tracking system. Practice has proved that the algorithm in the target tracking of dual DSP platform can detect and track field or predefined target in the image automatically, it overcomes the defect of traditional target tracking system.

\section{Introduction}

For requires a real-time tracking performance of the project at present, in addition to using a variety of traditional algorithm and improved algorithm, also must have a high speed hardware processing platform to run the algorithm. In view of the current real-time imaging tracking system requirements and the characteristics of algorithm, the system design the hardware processing platform with dual DSP for high-speed target tracking system, the traditional tracking algorithm and SVM algorithm run in the platform and application of the project successfully, from the experiment result shows, the hardware processing platform can meet the operation requirements of the SVM algorithm, the system calculate the target within $20 \mathrm{~ms}$ for the miss distance, meet the requirements of real-time and accuracy.

\section{The hardware structure and the performance requirements for the system}

System according to the characteristics of the SVM algorithm designed the target tracking system mainly composed of two hardware DSP processing module TMS320C6455, FPGA-XC2S200E storage control module, video signal processing module, high-speed adc module BT835 and data communication interface module, form the real-time target tracking of digital image processing platform, as shown in figure 1.

System USES BT835 for sampling PAL analog video signal, because the operation of DSP algorithm for gray image processing, so the system uses only high 8 signals output and send to the FPGA, at the same time, the row synchronization (AD_VREST and AD_HREST signals), the field synchronization (AD_FIELD signal) and sampling clock (AD_CLK signal) input to the FPGA, as interruption of FPGA and DSP processing and reference signal. The analog video signal of the system is sampled by BT835, the image preprocessing inside the FPGA as follows: (1) operated according to the external command of digital video signal,such as median filtering, edge detection 
and binarization pretreatment; (2) achieved the function of double buffer FIFO, stored the data of preprocessing image in the FIFO, waiting for the DSP to read; (3) achieved the character superposition, under the control of the DSP, produced the missing distance, the tracking window, the frame, the date code characters, etc by the field of different location. Two DSP read the images of the odd and even fields respectively, that is in a frame time of image storage processing, every DSP only deals with one of the image, and each DSP connected external MT47H64M16BT for the high-speed DDR memory. Computer communication using FPGA chip can realize the function of the PCI interface, so with the help of the FPGA parallel processing features, computer have no conflict with DSP each other through PCI interface to read/take, sampling images, make the system to achieve high performance.



Fig. 1 The real-time processing hardware platform system of Dual DSP

In order to meet the requirements of the project, the key performance indicators of the system: video signal unified CCIR, peak of the voltage used $1 \mathrm{v}$; processing the resolution of pixel for every images $768 \times 256$; measurement precision of 1 pixel; the data processing time is less than $20 \mathrm{~ms}$ of the tracking target for the center of gravity tracking and the matching measurement; the automatic acquisition time of long range gate is less than $60 \mathrm{~ms}$ within the whole field.

\section{SVM algorithm and other algorithms combined with the application of contrast}

If the image pixel value is directly used as the input data of the SVM trainer and classifier, the algorithm is too large, which leads to low tracking accuracy. Therefore, put forward three algorithms: (1) using Gabor wavelet to calculate the sample characteristics, and then use the energy function, principal component analysis (PCA) and AdaBoost algorithm to extract Gabor features; (2) by PCA, kernel principal component analysis (KPCA) and linear discriminant analysis (LDA) to reduce the dimensionality of the input data; (3) using Haar wavelet features extracted by AdaBoost, some of the typical characteristics of the three kinds of feature selection method combined with SVM for target tracking. 


\section{using the energy function, PCA and AdaBoost algorithm to select some Gabor features}

The system uses the UIUC car image database as the experimental object, and 500 positive samples and 1000 negative samples are selected to form the training sample (sample size is $24 \times 24$ ). In order to find the best parameters, the first 2000 samples were selected from the original training set as the new training set, and the other samples in the original training set were used as the validation set. Finally, the optimal parameters are searched by cross validation method.

The system experiment uses the energy function, the PCA algorithm and the AdaBoost algorithm to select the sample image and the Gabor filter convolution characteristic, then uses SVM to carry on the training classification, the classification result is shown in Table 1.

The experimental results show that the AdaBoost algorithm has more accurate than the energy function and PCA method to extract the Gabor wavelet.

Table 1 Experimental results of the Gabor feature selection methods

\begin{tabular}{|l|l|c|c|c|}
\hline \multicolumn{2}{|l|}{ Gabor feature selection algorithm } & energy function & PCA & AdaBoost \\
\hline \multirow{3}{*}{$\begin{array}{l}\text { Vehicle } \\
\text { target }\end{array}$} & Selected characteristic number & 150 & 150 & 150 \\
\cline { 2 - 5 } & $\begin{array}{l}\text { Linear kernel function SVM classification } \\
\text { accuracy }\end{array}$ & $71.3 \%$ & $73.2 \%$ & $85.6 \%$ \\
\cline { 2 - 5 } & $\begin{array}{l}\text { RBF kernel function SVM classification } \\
\text { accuracy }\end{array}$ & $73.5 \%$ & $76.8 \%$ & $93.4 \%$ \\
\hline
\end{tabular}

Figure 2 is based on the AdaBoost algorithm to select the Gabor features of the SVM tracking algorithm to track the results of aircraft targets, from the results of Figure 2 can be seen when the target rotation can still be accurately tracked. Because of the reduction of the number for the features of Gabor image feature extraction method based on AdaBoost algorithm, the time of target detection is greatly reduced.

\section{the dimensionality reduction on the input data by PCA, LDA and KPCA methods}

When the statistical method is used to solve pattern recognition, a lot of analysis or computation is not feasible in high dimensional space, but it is feasible in low dimensional space. Therefore, dimensionality reduction is very important in solving practical problems.

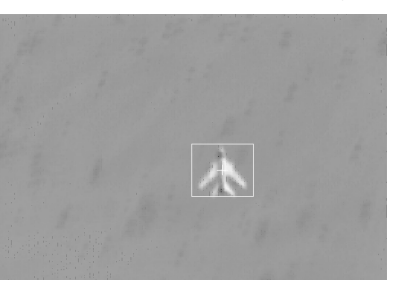

( a ) 0 th frame

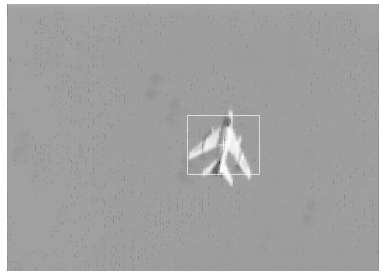

(d ) 150 th frame

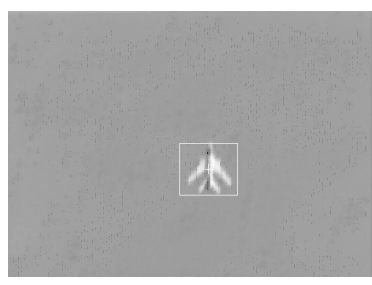

( b ) 50 th frame

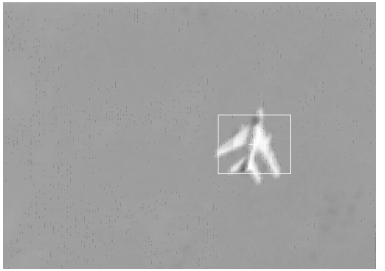

( e ) 200 th frame

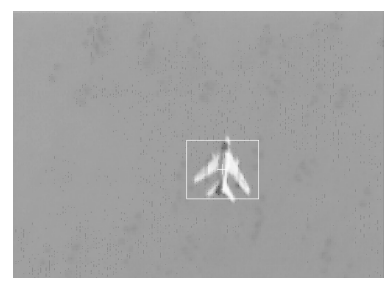

(c ) 100 th frame

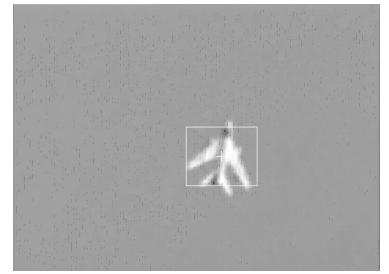

(f ) 250th frame

Fig.2 SVM tracking algorithm based on AdaBoost algorithm for Gabor target tracking results

The system uses PCA, KPCA and LDA method to calculate the characteristic matrix, the purpose is to reduce the dimension of the input samples, without loss of classification accuracy. 
The training samples and test samples are extracted from the feature matrix and input into the SVM for training. In the experiment, using the car image library, library is composed by 500 positive samples and 500 negative samples as training samples (sample size is $100 \times 40$ ), the test samples were selected 200 images with the same size of the training set and 100 images of different size and concentration of automotive training. The classification accuracy of the three methods using PCA, KPCA and LDA is shown in Table 2.

The experimental results show that KPCA is a generalization of the PCA method, and its accuracy is higher than that of PCA and LDA algorithm.

Table 2 SVM classification accuracy of PCA, KPCA and LDA's three feature extraction methods

\begin{tabular}{|l|l|r|r|l|}
\hline \multicolumn{2}{|l|}{ Selected characteristic number } & PCA & LDA & KPCA \\
\hline \multirow{2}{*}{$\begin{array}{l}\text { Vehicle } \\
\text { target }\end{array}$} & Linear kernel function SVM classification accuracy & $72.8 \%$ & $72.7 \%$ & $81.4 \%$ \\
\cline { 2 - 5 } & RBF kernel function SVM classification accuracy & $76.5 \%$ & $76.4 \%$ & $86.7 \%$ \\
\hline
\end{tabular}

\section{using AdaBoost to extract some representative image features}

Because the AdaBoost algorithm can select the optimal weak classifier effectively, then form a strong classifier, so SVM algorithm has good adaptability for small sample nonlinearity, therefore, the characteristics of using AdaBoost algorithm to extract the most representative, using SVM classification to improve the detection precision of target. The system combines AdaBoost algorithm to extract features, and then uses SVM learning and classification for target detection and tracking. Among them, in order to quickly detect the target, using the waterfall type detection algorithm, the SVM classifier in the first several layers use fewer features quickly exclude non target window large, using feature number characteristics of the trained classifier to detect the object accurately. Vehicle image is selected in the experiment, 150 kinds of positive samples and 450 negative samples are selected to form the training samples (sample size is $24 \times 24$ ), and Table 3 is the experimental result of the detection algorithm.

Table 3 Results of target detection

\begin{tabular}{|l|c|c|c|}
\hline Test sample type & Sample number & $\begin{array}{l}\text { Sample number of correct } \\
\text { classification }\end{array}$ & Correct detection rate ( \% ) \\
\hline Positive sample & 150 & 133 & 89.4 \\
\hline Negative sample & 450 & 412 & 91.5 \\
\hline
\end{tabular}

At the same time, the system will use several commonly image detection and tracking algorithm individually with SVM algorithm in the design of the dual DSP hardware platform, and compare the performance of the tracking system algorithm, as shown in Table 4. Seen from the table, SVM algorithm can output the target miss in every two frames in quantity by the system design of dual DSP platform. Can fully meet the real-time requirements of the project (the data processing time is less than $20 \mathrm{~ms}$ ), and the dual DSP system suitable for the running time more than $20 \mathrm{~ms}$ algorithm, so that the odd and even field respectively calculated by two DSP, and give full play to the advantages of the dual DSP.

Table 4 Time performance of dual DSP system

\begin{tabular}{|l|l|l|l|}
\hline Centroid & Mean-Shift & Circle projection correlation & SVM algorithm \\
\hline $3.6 \mathrm{~ms}$ & $7.4[\mathrm{~ms}]$ & $10.3[\mathrm{~ms}]$ & $17.2[\mathrm{~ms}]$ \\
\hline
\end{tabular}

\section{Conclusion}

From the experiments, can see that in the hardware platform of this system, the method of extracting some representative feature extraction by using AdaBoost combined with SVM has higher tracking precision. At the same time, in the process of detection and tracking, the cascade 
detection algorithm of the waterfall is used to accelerate the speed of target tracking, which makes it possible to achieve real-time performance.

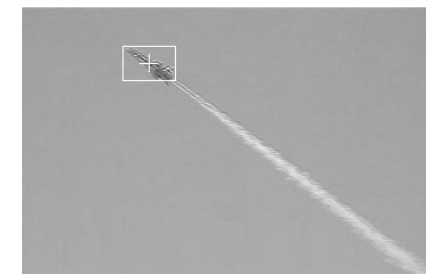

( a ) 5 th frame

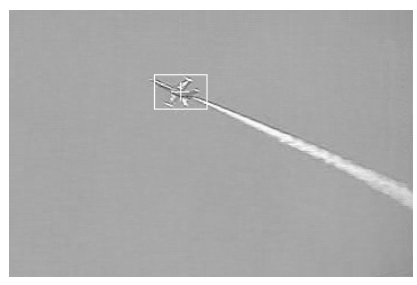

(d ) 80 th frame

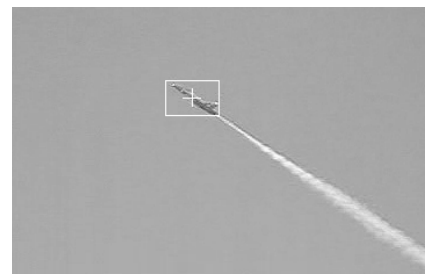

( b ) 20 th frame

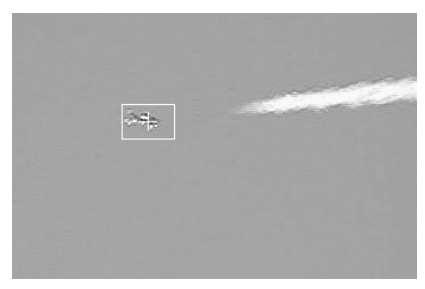

(e ) 130 th frame

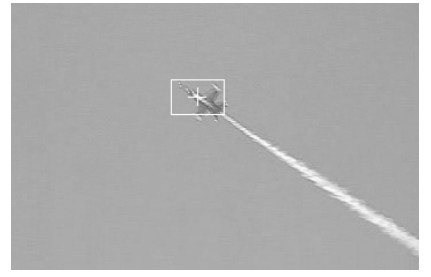

(c ) 50 th frame

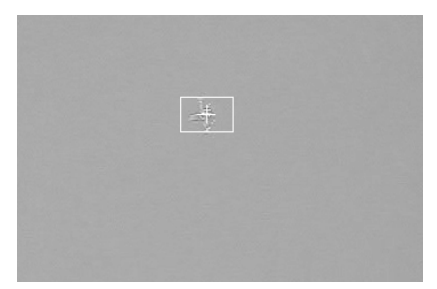

( f ) 180 th frame

Fig. 3 tracking results

\section{Acknowledgements}

This project is supported by the National Natural Science Foundation of China: 11375055.

\section{Reference}

[1] Huang Yong et al. A survey of multi class support vector machine [J], computer technology and automation, 2005-24 (4): 61-63

[2] Hu Jiajing, target detection system in the background modeling algorithm and DSP to achieve [D], Nanjing University of Posts and Telecommunications, 2012:37-41

[3] Fang Hui et al. Support vector machine training and classification algorithm [J], Journal of Daqing Normal University, 2009-3:85-88

[4] Yang Weilong and so on, support vector machine in the realization of DSP method [J], computer measurement and control, 2007-15 (1): 76-78 